Original Research Paper

\title{
Modification on PPS Sample Scheme with Replacement
}

\author{
${ }^{1}$ Ayed R.A. Alanzi, ${ }^{2}$ Naser A. Alodat and ${ }^{3}$ Ahmad M. Qazza \\ ${ }^{1}$ Department of Mathematics, College of Science and Human Studies at Hotat Sudair, \\ Majmaah University, Majmaah 11952, Saudi Arabia \\ ${ }^{2}$ Department of Mathematics, Jadara University, P.O. Box (733), postal code 21111, Irbid-Jordan \\ ${ }^{3}$ Department of Mathematics, Zarqa University, P.O. Box (132222), postal code 13132, Zarqa-Jordan
}

\author{
Article history \\ Received: 30-03-2019 \\ Revised: 30-05-2019 \\ Accepted: 18-06-2019 \\ Corresponding Author: \\ Ayed R.A. Alanzi \\ Department of Mathematics, \\ College of Science and Human \\ studies at Hotat Sudair, Majmaah \\ University, Majmaah 11952, \\ Saudi Arabia \\ Email: a.alanzi@mu.edu.sa, \\ auid403@hotmail.com
}

Abstract: In this paper we have developed an alternative estimator for the Probability Proportional to Size (PPS) with replacement sampling scheme when certain characteristics under study are positively correlated with the selection probability. An analogue to the well-known superpopulation model for finite population is also suggested, using which, we compare the proposed estimator with Hansen and Hurwitz estimator. Finally, an empirical investigation of the performance of the propose estimator has also been made.

Keywords: Correlation Coefficients, Probability Proportional to Size PPS with Replacement, Superpopulation Model

\section{Introduction}

Probability Proportional to Size (PPS) sampling is a method of sampling from finite population in which a size measure is available for each population unit before sampling and where the probability of selecting a unit is proportional to size.

Consider a finite population $U=\left(U_{1}, U_{2}, \ldots, U_{N}\right)$ consisting of $N$ distinct and identifiable units. Let $Y_{i}$ be the value of the study variable $y$ on the unit $U_{i}, i=1, \ldots$, $N$. In practice we wish to estimate the population total $Y=$ $\Sigma y_{i}$ from the $y$ values of the units drawn in a sample $\left(u_{1}\right.$, $\left.u_{2}, \ldots, u_{n}\right)$ with maximum precision. The easiest of the probability sampling scheme for drawing a sample $u$ is the Simple Random Sampling with Replacement (SRSWR) scheme for which an unbiased estimator of $y$ is given by:

$\widehat{T}_{w r}=\frac{N}{n} \sum_{i=1}^{n} y_{i}$

With variance is given by:

$V(\widehat{T} w r)=\frac{N}{n}\left[\sum_{i=1}^{N} y_{i}^{2}-\frac{Y^{2}}{N}\right]$.

Hansen and Hurwitz (1943) proposed the idea of sampling with Probability Proportional to Size (PPS) with replacement for positive correlated characteristics. This scheme was carried out as follows: One unit is selected at each of the $n$ draws. For each ith unit selected from the population, a selection probability is given by:

$$
p_{i}=\frac{x_{i}}{x}
$$

where, $x_{i}$ is the measure for $i$ th unit and:

$$
x=\sum_{i=1}^{n} x_{i}
$$

They gave the following estimator of population total $Y i$ as:

$$
\widehat{T}_{H H}=\frac{1}{n} \sum_{i=1}^{n} \frac{y_{i}}{p_{i}}
$$

With variance is given by:

$$
v\left(\widehat{T}_{H H}\right)=\frac{1}{n} \sum_{i=1}^{n} \frac{y_{i}^{2}}{p_{i}}-Y^{2}
$$

PPS sampling is expected to be more efficient than SRS sampling if the regression line of $y$ on $x$ passes through the origin. When it is not so, a transformation on the auxiliary variable can be made so that the PPS sampling with modified sizes becomes more efficient. Reddy and Rao (1977) suggested that the sample be selected by probability proportional to revised sizes scheme and with replacement, the revised sizes are obtained through a location shift in the auxiliary variable as:

$$
X^{*}=X_{i}+\left(\frac{1}{L}-1\right) \bar{X} \quad 0<L<1
$$


However, only one measure of size is usually used in selecting primary sampling units in PPS scheme. In contrast, it may sometimes happen that some of these study variables are poorly but positively correlated with selecting probabilities, thereby reducing the existing estimator inadequate. An alternative estimator was proposed by Rao (1966). Bansal and Singh (1985), Amahia et al. (1989), Enang and Amahia (2012) and others have proposed an estimator for characteristics that are poorly correlated with selecting probabilities.

Sahoo et al. (1994) suggested a simple transformation of the auxiliary variable where the correlation between study variable and auxiliary variable is highly negative.

Bedi and Rao (1997) gave a new direction in determining estimator of population total under the PPSWR sampling scheme when the correlation between the auxiliary variable and study variable is negative.

In this paper we suggested a simple transformation on $x$ to $x^{*}$ such that $x^{*}=\left(x+x_{i}\right)$.

We have also obtained the condition under which the proposed estimator will be more efficient than Hansen and Hurwitz (1943) estimator. The condition has been derived under the superpopulation model given below.

\section{The Superpopulation Model}

Let $y_{i}$ and $p i$ denote the value of characteristics $y$ and the relative measure of size $\mathrm{p}$ for the $i$ th $(i=1,2, \ldots, N)$ unit in the population, respectively. A general superpopulation model suitable for our case is:

$$
y_{i}=B p_{i}+e_{i}, i=1,2, \ldots, N,
$$

where, $e_{i}$ are the errors such that:

$$
\begin{aligned}
& E\left(e_{i} / p_{i}\right)=0, \\
& E\left(e_{i}^{2} / p_{i}\right)=\sigma^{2} p_{i}^{g}, \\
& \sigma^{2}>0, g \geq 0, \\
& E\left(e_{i} e_{j} / p_{i} p_{j}\right)=0,
\end{aligned}
$$

where, $E($.$) denote the average overall finite population$ that can be drawn from the super population. There are many papers in which the supper population model is successfully used for the purpose of comparing the different sample strategies, see, Godambe (1955), Brewer (1963), Rao (1966), Hanurav (1976) and many others.

PPS sampling is considered to be more efficient than SRS sampling if the regression line of $y$ on $x$ passes through the origin Raj (1954). When it is not so a transformation on the auxiliary variable can be made so that the PPS sampling with modified sizes become more precise.

\section{Suggested Estimator}

Suppose that the auxiliary variable $x>0$ has a positive correlation with study variable $y$. Then we suggest the following transformation on $x$ to $x^{*}$ such that $x^{*}=(x+$ $\left.x_{i}\right), i=1,2, \ldots, N$. Naturally $x^{*}$ is greater than zero. Further, we can easily see that correlation between $y$ and $x^{*}$ is also positive. Hence the modified probabilities of selection become:

$p_{i}^{*}=\frac{1+p_{i}}{N+1} i,=1,2, \ldots, N$

Then the estimator of the population total $Y$ is give by:

$$
\widehat{Y}_{p}=\frac{1}{n} \sum_{i=1}^{n} \frac{y_{i}}{p_{i}^{*}}
$$

\section{The Variance and its Expected Value of the Suggested Estimator}

It is well known that the variance of the usual estimator $\widehat{T}_{H H}$ is given by:

$v\left(\widehat{T}_{H H}\right)=\frac{1}{n}\left[\sum_{i=1}^{N} \frac{y_{i}^{2}}{p_{i}}-\left(\sum_{i=1}^{n} y_{i}\right)^{2}\right]$

The corresponding variance of the estimator due to Rao is obtained by:

$v\left(\widehat{T}_{R}\right)=\frac{N^{2}}{n}\left[\sum_{i=1}^{N} y_{i}^{2} p_{i}-\left(\sum_{i=1}^{N} y_{i} p_{i}\right)^{2}\right]$

The variance of proposed estimator is obtain by replacing $p_{i}$ by $p_{i}^{*}$ in (7) and is given by:

$$
v\left(\widehat{Y}_{p}\right)=\frac{1}{n}\left[\sum_{i=1}^{N} \frac{y_{i}^{2}}{p_{i}^{*}}-\left(\sum_{i=1}^{N} y_{i}\right)^{2}\right]
$$

\section{Robustness Estimator}

Now, we state two lemmas, which are useful for estimator's comparisons.

\section{Lemma 1}

Royall (1970) Let $0 \leq b_{1} \leq b_{2} \leq \ldots \leq b_{m}$ and $c_{1} \leq c_{2} \leq \ldots \leq c_{m}$ satisfying:

$$
\sum_{i=1}^{m} c_{i} \geq 0
$$

then:

$$
\sum_{i=1}^{m} b_{i} c_{i} \geq 0
$$




\section{Lemma 2}

Let $b_{1} \geq b_{2} \geq \ldots \geq b_{m} \geq 0$ and $c_{1} \geq c_{2} \geq \ldots \geq c_{m}$ satisfy:

$$
\sum_{i=1}^{m} c_{i} \geq 0
$$

then:

$$
\sum_{i=1}^{m} b_{i} c_{i} \geq 0
$$

\section{Theorem}

Under the superpopulation model, the sufficient condition that $\widehat{T}_{H H}$ has smaller expected variance than $\hat{Y}_{p}$ is:

$$
g \geq 1+\frac{p_{i}}{1+p_{i}}
$$

\section{Proof}

Under the superpopulation model the expected variance of $\widehat{T}_{H H}$ and $\widehat{Y}_{p}$ are respectively given by:

$$
n E\left(v\left(\widehat{T}_{H H}\right)\right)=\sigma^{2} \sum_{i=1}^{N} p_{i}^{g}\left(1-p_{i}\right)
$$

and:

$$
n E\left(v\left(\widehat{Y}_{p}\right)\right)=B^{2}\left[\sum_{i=1}^{N} \frac{p_{i}^{2}}{p_{i}^{*}}-\left(\sum_{i=1}^{N} p_{i}\right)^{2}\right]+\sigma^{2} \sum_{i=1}^{N} p_{i}^{g}\left(\frac{1}{p_{i}^{*}}-1\right) .
$$

The difference between them can be written as:

$$
\begin{aligned}
& n\left[E\left(v\left(\widehat{Y_{p}}\right)\right)-E\left(v\left(\widehat{T_{H H}}\right)\right)\right]=B^{2}\left[\sum_{i=1}^{N} \frac{p_{i}^{2}}{p_{i}^{*}}-\left(\sum_{i=1}^{N} p_{i}\right)^{2}\right] \\
& +\sigma^{2} \sum_{i=1}^{N} p_{i}^{g-1}\left(\frac{p_{i}-p_{i}^{*}}{p_{i}^{*}}\right)=B^{2}\left[\sum_{i=1}^{N} \frac{p_{i}^{2}}{p_{i}^{*}}-\left(\sum_{i=1}^{N} p_{i}\right)^{2}\right] \\
& +\sigma^{2} \sum_{i=1}^{N} p_{i}^{g-1} \frac{N p_{i}-1}{(N+1) p_{i}^{*}}=B^{2}\left[\sum_{i=1}^{N} \frac{p_{i}^{2}}{p_{i}^{*}}-\left(\sum_{i=1}^{N} p_{i}\right)^{2}\right] \\
& +\sigma^{2} \sum_{i=1}^{N} p_{i}^{g-1} \frac{N p_{i}-1}{\left(1+p_{i}\right)}=B^{2}\left[\sum_{i=1}^{N} \frac{p_{i}^{2}}{p_{i}^{*}}-\left(\sum_{i=1}^{N} p_{i}\right)^{2}\right]+\sigma^{2} \sum_{i=1}^{N} b_{i} c_{i},
\end{aligned}
$$

where, $c_{i}=\left(N p_{i}-1\right)$ and $b_{i}=\frac{p_{i}^{g-1}}{1+p_{i}}$. Note that, the above first term of the above expression is always positive. For the second term we observe that $\sum c_{i}=0$ and $c_{i}$ is an increasing function of $i$. So in view Royall's lemma 1 it can be shown that $\sum b_{i} c_{i}>0$ provided $b_{i}$ is also increasing function of $p_{i}$. By deriving $b_{i}$ with respect to $p_{i}$ we get that the sufficient condition that makes $T_{H H}$ has smaller variance than $\hat{Y}_{P}$ is:

$$
g \geq 1-\frac{p_{i}}{1+p_{1}^{\prime}}
$$

Hence the theorem is proved.

\section{Empirical Study}

To study the behavior of the estimator $\hat{Y}_{P}$ with the conventional estimator $\widehat{T}_{H H}$, we consider the five population $A, B, C, D$ and $E$, details of which are given in Table 1. The population $A, B$ and $C$ are the same as the three population of the Yates and Grundy (1953).

Table 1: Populations under

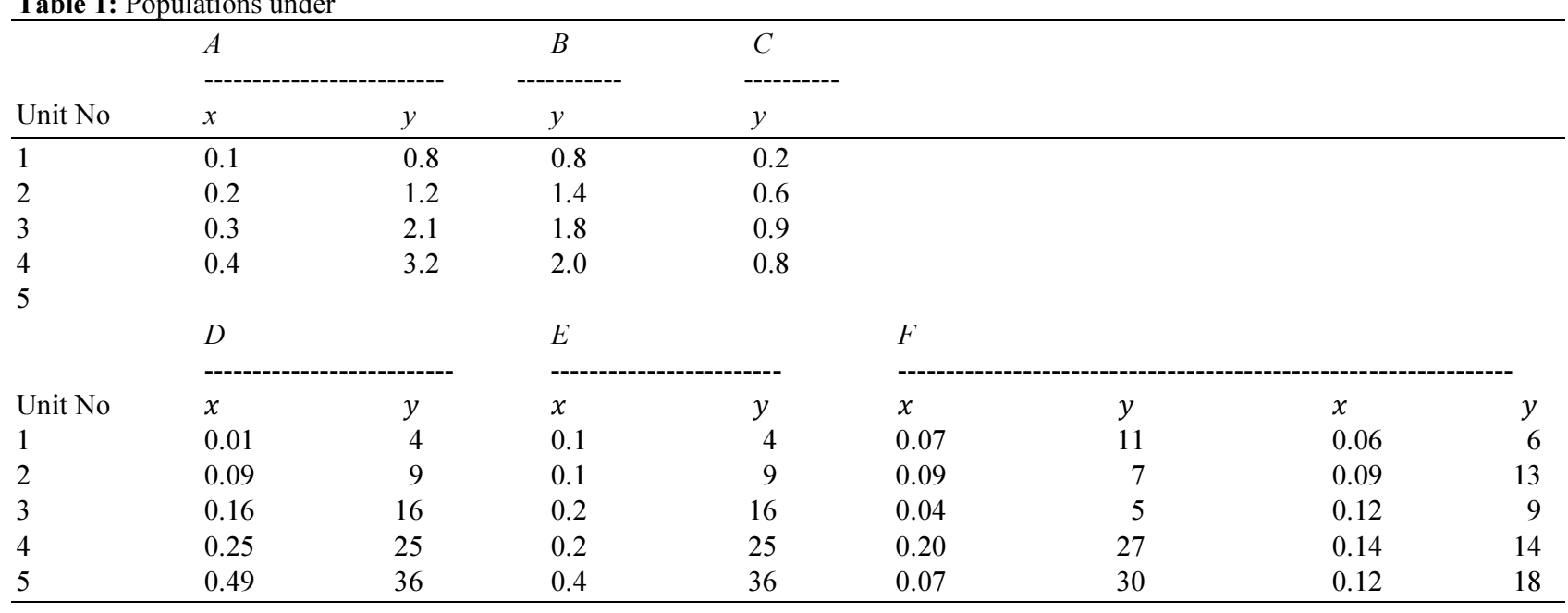


Table 2: Variance of the estimator

\begin{tabular}{lrrrr}
\hline POP & $\widehat{T}_{W R}$ & $\widehat{T}_{H H}$ & $\widehat{T}_{R}$ & $\hat{Y}_{p}$ \\
\hline A & 6.815 & 0.305 & 6.384 & 4.576 \\
B & 1.680 & 0.500 & 1.104 & 0.911 \\
C & 0.575 & 0.125 & 0.337 & 0.416 \\
D & 498.000 & 1842.000 & 890.902 & 569.108 \\
E & 498.000 & 1229.200 & 765.200 & 597.871 \\
F & 3350.000 & 3708.440 & 3221.620 & 3151.820 \\
\hline
\end{tabular}

Table 3: Percentage variances relative to the suggested estimator

\begin{tabular}{lcccc}
\hline POP & $\widehat{T}_{W R}$ & $\widehat{T}_{H H}$ & $\widehat{T}_{R}$ & $\hat{Y}_{p}$ \\
\hline $\mathrm{A}$ & 149.12 & 6.67 & 139.5 & 1 \\
$\mathrm{~B}$ & 184.41 & 54.88 & 121.18 & 1 \\
$\mathrm{C}$ & 138.22 & 30.05 & 81.01 & 1 \\
$\mathrm{D}$ & 87.51 & 323.66 & 156.5 & 1 \\
$\mathrm{E}$ & 83.30 & 205.61 & 129.99 & 1 \\
$\mathrm{~F}$ & 106.23 & 117.65 & 102.21 & 1 \\
\hline
\end{tabular}

Whereas population $D$ is of Stuart (1986). The population $E$ is of Stuart (1986) and population $F$ is of Amahia et al. (1989).

Table 2 gives the variances of the proposed estimator $\hat{Y}_{p}$ with the conventional estimators $\widehat{T}_{W R}, \widehat{T}_{H H}$ and $\hat{T}_{R}$ for $n=2$.

Table 3 gives the percentages efficiency of the proposed estimator $\hat{Y}_{p}$ with the conventional estimators $\widehat{T}_{W R}, \widehat{T}_{H H}$ and $\hat{T}_{R}$.

\section{Conclusion}

Table 3 give the percentage efficiency of the proposed estimators $\hat{Y}_{p}$ with the conventional estimator $\widehat{T}_{W R}, \widehat{T}_{H H}$ and $T_{R}$ for $n=2$.

It is clear from Table 3 that the proposed estimator $\widehat{Y}_{p}$ performed better in populations $A$ and $B$ than $\widehat{T}_{W R}$, $\widehat{T}_{R}$. In population $C$ it is clear that the proposed estimator $\hat{Y}_{p}$ performed better than $\hat{T}_{W R}$. But in population $D$ and $E$ the proposed estimator performed than $\hat{T}_{H H}$ and $\hat{T}_{R}$.

We can see that in population $F$ the proposed estimator $\hat{Y}_{p}$ performed better than the $\widehat{T}_{W R}, \widehat{T}_{H H}$ and $\widehat{T}_{R}$.

\section{Acknowledgement}

The authors gratefully acknowledge with thanks the very thoughtful and constructive comments and suggestions of the Editor-in-Chief and the reviewers which resulted in much improved paper.

\section{Author's Contributions}

Authors contributed to the same extent to all the process of preparing and developing the manuscript since we operate as a group.

\section{Ethics}

This article is original and contains unpublished material. The corresponding author confirms that all of the other authors have read and approved the manuscript and there are no ethical issues involved.

\section{References}

Amahia, G.N., Y.P. Chaubey and T.J. Rao, 1989. Efficiency of a new estimator in PPS sampling for multiple characteristics. J. Stat. Plann. Inference, 21: 75-84.

Bansal, M.L. and R. Singh, 1985. An alternative estimator for multiple characteristics in PPS sampling. J. Statist. Plann. Inference, 11: 313-320.

Bedi, P.K. and T.J. Rao, 1997. PPS method of estimation under a transformation. J. Indian Soc. Agar. Stat.

Brewer, K.R.W., 1963. A method of systematic sampling with unequal probabilities. Aust. J. Stat., 5: $5-13$.

Enang, E. I. and G.N. Amahia, 2012. A class of alternative estimators in probability proportional to size sampling with replacement for multiple characteristics. J. Math. Res., 4: 66-77.

Godambe, V.P. 1955. A unified theory of sampling from finite populations. J. Roy. Stat. Soc., 17: 269-278.

Hansen, M.H. and W.N. Hurwitz, 1943. On the theory of sampling from finite populations. Ann. Math. Stat., 14: 333-362. 
Hanurav, T.V., 1976. Optimum utilization of auxiliary information: $\pi p s$ sampling of two units from a straturn. Jout. Roy. Stat. Soc., 29: 374-391.

Raj, D., 1954. On the sampling with probabilities proportional to size. Ganits, 5: 175-182.

Rao, J.N.K., 1966. On the relative efficiency of some estimators in PPS sampling for multiple characteristics. Sankhya A, 28: 61-70.

Reddy, V.N and T.J. Rao, 1977. Modified PPS method of estimation. Sankhya, 39: 185-197.

Royall, R.M., 1970. On finite population sampling theory undercertain linear regression models. Biometrika, 57: 377-387.
Sahoo, J., L.N. Sahoo and Mohanty, 1994. Unequal probability sampling using a transformed variable. Metron, 52: 71-83.

Stuart, A., 1986. Location shifts in sampling with unequal probabilities. J. Roy, Stat. Soc., 149: 169-174.

Yates, F. and P.M. Grundy, 1953. Selection without replacement from within strata with probability proportional to size. J. Roy. Stat. Soc. Ser., 5: 253-261. 